Quim. Nova, Vol. 34, No. 2, 196-199, 2011

\title{
RELACIÓN ENTRE LA ENTALPÍA DE INMERSIÓN DE MONOLITOS DE CARBON ACTIVADO Y PARAMETROS TEXTURALES
}

\author{
Diana P. Vargas y Liliana Giraldo Gutiérrez \\ Departamento de Química, Facultad de Ciencias, Universidad Nacional de Colombia, Av. Carrera 30, 45 -03, Bogotá, Colombia \\ Juan Carlos Moreno* \\ Departamento de Química, Facultad de Ciencias, Universidad de los Andes, Carrera 1, 18 A 10, Bogotá, Colombia
}

Recebido em 4/1/10; aceito em 27/8/10; publicado na web em 24/11/10

\begin{abstract}
RELATION BETWEEN IMMERSION ENTHALPY OF ACTIVATED CARBON MONOLITHS AND TEXTURAL PARAMETERS. In this study, Disc and honeycomb-shaped activated carbon monoliths were obtained using as a precursor coconut shell, without the use of any binder. Textural characterization was performed by adsorption of $\mathrm{N}_{2}$ at $77 \mathrm{~K}$ and immersion calorimetry into benzene. The experimental results showed that the activation with zinc chloride produces a wide development of micropores, yielding micropore volumes between 0,38 and $0,79 \mathrm{~cm}^{3} \mathrm{~g}^{-1}$, apparent BET surface area between 725 and $1523 \mathrm{~m}^{2} \mathrm{~g}^{-1}$ and immersion enthalpy between 73,5 and 164,2 $\mathrm{J} \mathrm{g}^{-1}$. Were made comparisons between textural parameters and energy characteristics.
\end{abstract}

Keywords: carbon monolith; chemistry activation; immersion enthalpy.

\section{INTRODUCCIÓN}

Durante muchos años el hombre se ha beneficiado de su ambiente y ha causado un gran impacto sobre el equilibrio natural de los ecosistemas, hasta llegar a amenazar la sostenibilidad del planeta. Por esta razón, ha surgido la necesidad de crear nuevas tecnologías que contribuyan a disminuir la cantidad de contaminantes en fase acuosa y gaseosa. Una de estas nuevas tecnologías, es el carbón activado, un material que se prepara artificialmente para que presente propiedades adsorbentes. ${ }^{1}$ A lo largo del tiempo se han desarrollado diferentes formas de presentación de este material: gránulos, polvo, pellets, telas, fibras y monolitos, estos últimos presentan propiedades que los diferencia de las formas convencionales de carbón. La palabra monolito es "una sola piedra" hace referencia a estructuras compactas como los discos y honeycombs estos últimos son estructuras unitarias atravesadas longitudinalmente por canales paralelos, estas nuevas estructuras, presentan bajos valores de pérdida de carga al paso de los gases a tratar, excelentes propiedades mecánicas y reducen las limitaciones generadas por fenómenos de difusión interna. ${ }^{2}$ La importancia de los monolitos de carbón activado radica en que gracias a las propiedades que presentan son múltiples sus aplicaciones en el campo de la catálisis, adsorción y almacenamiento de gases entre otros. ${ }^{3,4}$ De esta forma se ha comprobado mediante estudios la capacidad de estas estructuras para almacenar gas natural gracias a que la forma monolítica aumenta la densidad y maximiza el volumen de gas natural adsorbido por volumen del recipiente, adicionalmente en estudios recientes se han probado como soportes para diferentes metales que se usan en diversos proceso catalíticos, así mismo se han utilizado como electrodos en supercapacitores. ${ }^{5,6} \mathrm{La}$ síntesis de monolitos de carbón, utilizables como soportes o adsorbentes directos, surge como una alternativa a las problemáticas ambientales y constituye un campo de investigación interesante.

Por otro lado, los monolitos sintetizados, deben ser caracterizados para establecer sus propiedades y posibles aplicaciones. Las técnicas de caracterización de sólidos han sido desde hace varias

*e-mail: jumoreno@uniandes.edu.co décadas una de las herramientas más importantes con que cuentan los investigadores de diversas áreas. La caracterización de sólidos es de importancia en actividades industriales como, la manufactura de los catalizadores, y el desarrollo de catalizadores más resistentes a la desactivación, activación y regeneración. En la investigación de un sólido, el primer paso es averiguar la naturaleza de la superficie del sólido, entre estas se tiene: estructura porosa, área superficial y su naturaleza química. De esta forma resulta útil la adsorción física de gases, específicamente $\mathrm{N}_{2}$ A $77 \mathrm{~K}$ y $\mathrm{CO}_{2}$ a $273 \mathrm{~K}$ para establecer el área superficial (BET), volumen de poro entre otros parámetros, ${ }^{7}$ así mismo se suele utilizar la calorimetría de inmersión. ${ }^{8,9}$

En este trabajo se estudian seis muestras de monolitos tipo disco y honeycombs, sobre las que se determinan isotermas de adsorción y calorimetrías de inmersión en benceno, con los datos obtenidos se calcula el área BET, volumen de microporos, energía característica y entalpía de inmersión de cada muestra.

\section{PARTE EXPERIMENTAL}

\section{Obtención de los monolitos}

La cáscara de coco se trituró y se tamizó utilizando para la síntesis un tamaño de partícula de $38 \mu \mathrm{m}$. El precursor, se impregno con $\mathrm{ZnCl}_{2}$ (1 gr de precursor por $2 \mathrm{~mL}$ de solución) por $7 \mathrm{~h}$ y a 358 $\mathrm{K}$. Luego se sometió a secado a $383 \mathrm{~K}$ por aproximadamente $2 \mathrm{~h}$. A continuación se llevó a una prensa uniaxial, en donde se hace el conformado por prensado a $423 \mathrm{~K}$. Estas estructuras se carbonizan en un horno horizontal, a una velocidad de calentamiento lineal de 273 $\mathrm{K} \mathrm{min}^{-1}$ hasta alcanzar una temperatura de $773 \mathrm{~K}$ permaneciendo allí durante $2 \mathrm{~h}$, con un flujo de $\mathrm{N}_{2}$ de $85 \mathrm{~mL} \mathrm{~min}^{-1}$. Finalmente, se lavan con ácido clorhídrico $0,1 \mathrm{~mol} \mathrm{~L}^{-1}$ y agua destilada hasta $\mathrm{pH}$ neutro para eliminar los restos del agente químico usado en la impregnación. ${ }^{10-14}$

Se utilizaron soluciones de 20, 32 y $48 \%$ de $\mathrm{ZnCl}_{2}$, para preparar las series de monolitos simbolizadas mediante las letras MD para discos y MH para honeycombs, seguido de la concentración utilizada para la serie, manteniendo constantes las demás condiciones. 


\section{Caracterización de las estructuras}

Todos los monolitos de carbón activado fueron caracterizados por adsorción física de $\mathrm{N}_{2}$ a $77 \mathrm{~K}$ usando un equipo Quantachrome, Autosorb 3-B. El volumen de microporo fue calculado mediante la aplicación de la ecuación de Dubinin-Radushkevich y el área superficial se obtuvo mediante el método BET. Las muestras fueron también caracterizadas mediante calorimetría de inmersión en benceno $(0,37$ $\mathrm{nm}$ ) usando un equipo tipo Calvet. ${ }^{8}$

\section{RESULTADOS Y DISCUSIÓN}

Los monolitos obtenidos presentan un diámetro de $1,5 \mathrm{~cm}$ con una altura de $8 \mathrm{~mm}$, en los monolitos tipo honeycombs el tamaño de los canales transversales es de $3 \mathrm{~mm}$. Las estructuras obtenidas se muestran en la Figura 1.

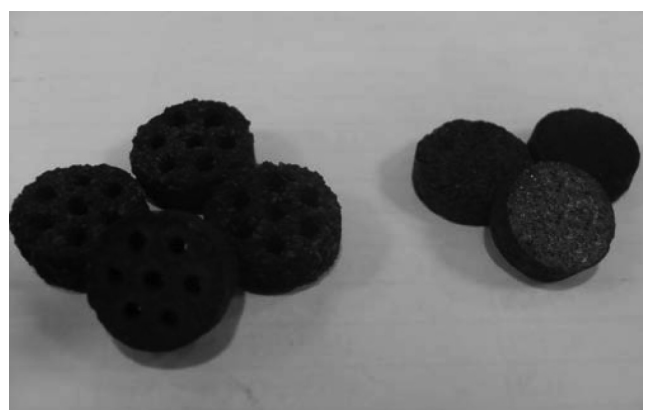

Figura 1. Estructuras obtenidas: a) disco, b) honeycomb

Luego de obtener los monolitos, estos fueron caracterizados mediante adsorción de nitrógeno a $77 \mathrm{~K}$. Se obtuvieron isotermas de adsorción Tipo I para todos los monolitos disco y honeycomb, lo cual indica que las condiciones experimentales de impregnación, prensado y carbonización empleadas en la preparación permiten la obtención de sólidos microporosos. El volumen de nitrógeno adsorbido estuvo entre 500 y $420 \mathrm{~cm}^{3} \mathrm{~g}^{-1}$ para el disco y el honeycomb respectivamente, que comprueba la capacidad de adsorción en estos sólidos.

Así mismo se utilizó el modelo BET para determinar el área superficial de los sólidos. Para la estimación del área superficial específica se han propuesto teorías en las que se supone el cubrimiento de la superficie del sólido por capas de adsorbato, organizadas en forma de un empaquetamiento cercano tal que la suma de las áreas transversales de las moléculas adsorbidas por unidad de masa representarían un estimado del área superficial. El modelo BET, fue desarrollado a partir del propuesto por Langmuir permitiendo extender el análisis a la adsorción en multicapa. De acuerdo con el modelo, las moléculas adsorbidas en una capa actúan como sitios de adsorción para la siguiente capa y a cualquier presión por debajo de la presión de saturación hay fracciones de la superficie cubiertas por $0,1,2 \ldots \mathrm{n}$ capas de moléculas adsorbidas. ${ }^{15}$ En la Figura 2 se muestra la aplicación del modelo BET para las muestras, se observa que se obtienen tendencias lineales en todos los casos, además existe una correlación entre el grado de inclinación de la línea con el área superficial, así entre mayor inclinación mayor área superficial.

Así, los monolitos de carbón activado preparados presentan áreas superficiales entre 725 y $1523 \mathrm{~m}^{2} \mathrm{~g}^{-1} \mathrm{y}$ volumen de microporo entre 0,38 y $0,79 \mathrm{~cm}^{3} \mathrm{~g}^{-1}$ como se presenta en la Tabla 1 , resultados que son satisfactorios teniendo en cuenta los reportes recientes en la preparación de estos materiales en los que se han obtenido resultados de área BET entre 500 y $2500 \mathrm{~m}^{2} \mathrm{~g}^{-1} \mathrm{y}$ un volumen de microporo entre 0,2 y $1,4 \mathrm{~cm}^{3} \mathrm{~g}^{-1} \cdot{ }^{10-14}$

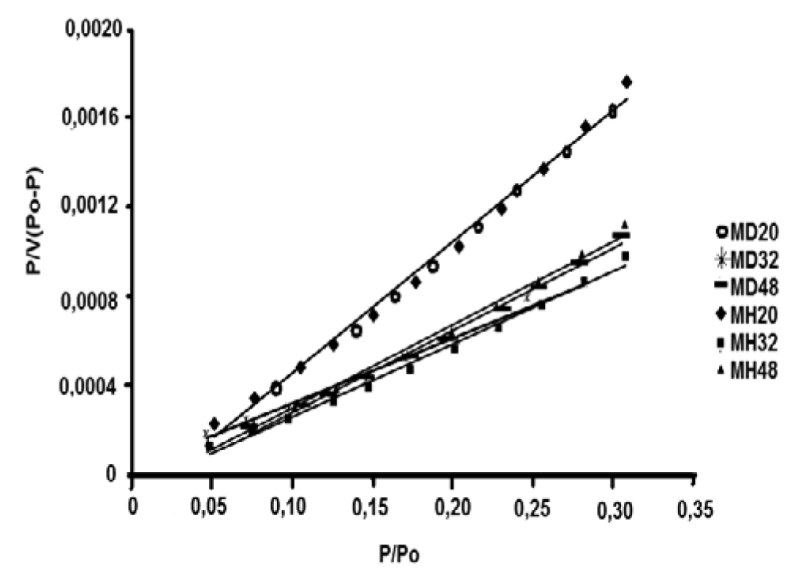

Figura 2. Modelo BET aplicado a los datos de isotermas de adsorción de nitrógeno de los monolitos

Tabla 1. Características de los monolitos de carbón

\begin{tabular}{ccccc}
\hline Muestra & $\begin{array}{c}\mathrm{S}_{\text {BET }} \\
\left(\mathrm{m}^{2} \mathrm{~g}^{-1}\right)\end{array}$ & $\begin{array}{c}\mathrm{S}_{\text {acc }} \\
\left(\mathrm{m}^{2} \mathrm{~g}^{-1}\right)\end{array}$ & $\begin{array}{c}\text { Volumen de } \\
\text { microporo } \\
\left(\mathrm{cm}^{3} \mathrm{~g}^{-1}\right)\end{array}$ & $\begin{array}{c}-\Delta \text { Hinm }_{\text {de la molecula sonda }} \\
\left(\mathrm{J} \mathrm{g} \mathrm{g}^{-1}\right)\end{array}$ \\
\hline $\begin{array}{c}\text { Negro de } \\
\text { carbono }\end{array}$ & 30 & 30,6 & ---- & 3,36 \\
MD20 & 821 & 820 & 0,45 & 90,2 \\
MD32 & 1523 & 1490 & 0,79 & 164,2 \\
MD48 & 1206 & 1200 & 0,65 & 132,1 \\
MH20 & 726 & 759 & 0,38 & 73,5 \\
MH32 & 1315 & 1302 & 0,72 & 134,1 \\
MH48 & 1170 & 1050 & 0,62 & 117,5 \\
\hline
\end{tabular}

En la Figura 3, se observa que el rendimiento del proceso de carbonización aumenta, conforme aumenta el radio de impregnación; este hecho se ratifica con el TGA del precursor realizado, pero omitido en el escrito, ya que a mayor concentración del agente impregnante mayor masa residual disponible para la carbonización debido a una mayor cantidad de materia volátil retenida. El calentamiento que se hace durante el prensado reduce la perdida de materia volátil, aumentando la resistencia y guardando todos los estados de impregnación. Durante el tratamiento térmico el impregnante actúa como un agente deshidratante favoreciendo la liberación de $\mathrm{H}_{2}$ y $\mathrm{O}_{2}$ al interior en forma de $\mathrm{H}_{2} \mathrm{O}$ y COx o hidrocarbonos. $\mathrm{Al}$ mismo tiempo, inhibe la perdida de materia volátil y alquitranes que fijan el carbón y hacen que el sólido mejore su rendimiento. Este agente penetra en el interior de las partículas produciendo una fragmentación parcial de la celulosa y otros biopolímeros de estructura vegetal tales como hemicelulosa y lignina; las reacciones siguientes son la deshidratación y condensación permitiendo que más compuestos aromáticos se produzcan con apariencia de alquitranes, los cuales permanecen en la superficie de la partícula impregnada y actúan como aglomerante. Gracias a esto no es necesario el uso de ningún material que una las partículas del precursor, es decir la porosidad no se ve afectada por la presencia de binder después de la carbonización. Gracias a la impregnación del precursor con $\mathrm{ZnCl}_{2}$, la masa es deformable con la presión, lo que permite la reducción del espacio entre partículas y el aumento de la densidad. ${ }^{11-14}$

En la Figura 4 se presenta la relación entre el área BET de los monolitos y el volumen de microporo, se observa una tendencia lineal para la serie discos y honeycomb, hay un aumento del área BET con el aumento de volumen de poro, este resultado es interesante ya que 


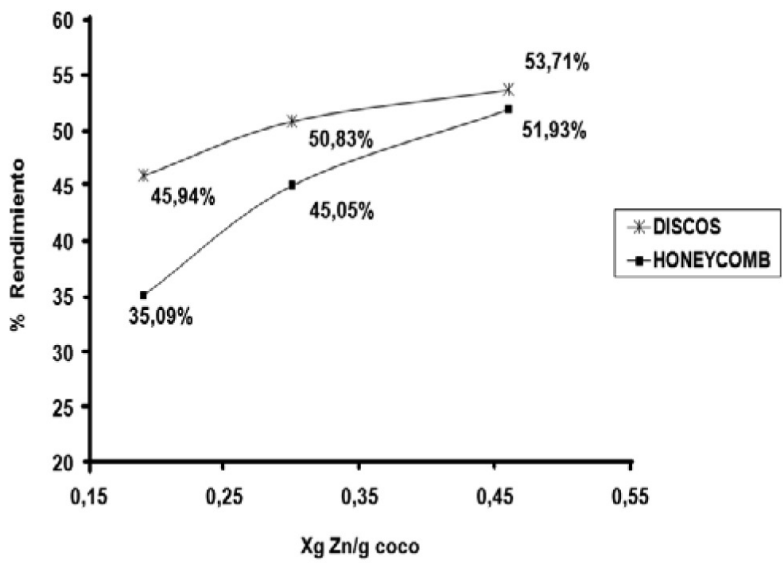

Figura 3. Relación entre el rendimiento y el rango de impregnación de los monolitos

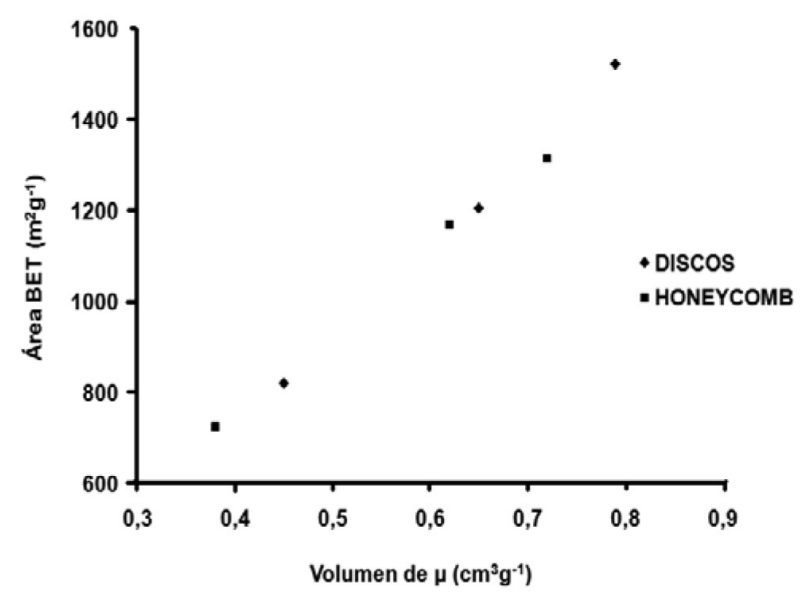

Figura 4. Relación entre el área BET y el volumen de microporo de los monolitos

muestra una buena correlación entre dos modelos con consideraciones diferentes, el modelo BET y el modelo DR.

En la Tabla 1, se muestran los valores de entalpía de inmersión en benceno. Se calculó el área por aproximación del área superficial accesible, para esto se tomó como referencia un sólido no poroso $\mathrm{Ne}$ gro de Carbono de área superficial $30 \mathrm{~m}^{2} / \mathrm{g}$, para calcular las entalpía especifica de la molécula sonda se la cual fue de $0.110-\Delta \mathrm{h}_{\text {especifica de la }}$ molécula $\left(\mathrm{m}^{2} \mathrm{~J}^{-1}\right),{ }^{9}$ comparándolas con los datos de las áreas calculadas por las isotermas de adsorción de $\mathrm{N}_{2}$ se puede decir que la desviaciones no son tan significativas para las muestras, teniendo en cuenta que cada modelo tiene sus propias consideraciones y no hay un modelo absoluto para calcular áreas superficiales. Las consideraciones de este modelo es que el líquido solo moja la superficie y no se considera el llenado de los microporos. En la Figura 5, se aprecia la gráfica propia de la calorimetría de inmersión realizada para la muestra con mayor y menor entalpía de inmersión, para los monolitos tipo honeycomb, el primer pico corresponde al contacto entre el sólido y el benceno, el otro pico es la calibración eléctrica hecha. El calor generado en la inmersión de la muestra es proporcional al área bajo la curva, de la relación del potencial termométrico en función del tiempo.

La Figura 6 presenta la relación entre la entalpía de inmersión en benceno y el área BET de los monolitos, se observa que a mayor área BET hay una mayor entalpía, siendo este el comportamiento esperado ya que hay una mayor superficie dispuesta a interactuar con el adsorbato, las entalpías de inmersión de los discos son mayores que las de

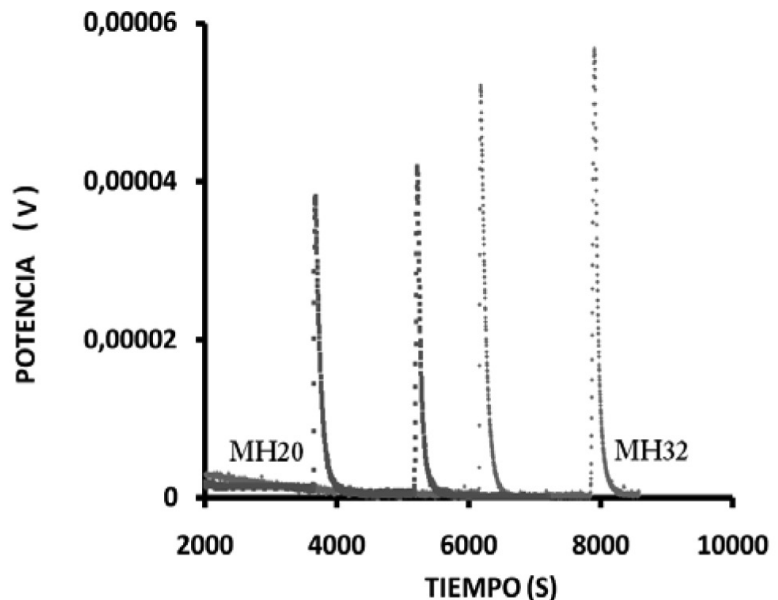

Figura 5.Calorimetrias de inmersión en benceno

los honeycomb debido a que estos sólidos tienen mayor porosidad y área superficial. Estos resultados muestran que la calorimetría de inmersión es una técnica de caracterización complementaria, que permite establecer correlaciones entre parámetros texturales de los sólidos y características energéticas, lo cual resulta útil para el estudio de la capacidad de adsorción de sólidos porosos.

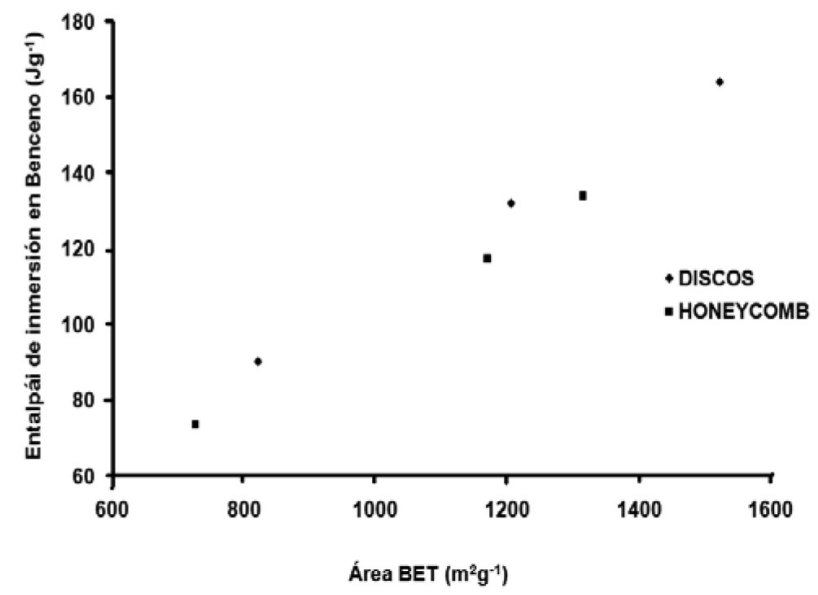

Figura 6. Relación entre la entalpía de inmersión en benceno y el área BET de los monolitos

Adicionalmente se determinó el producto EoWo, la Figura 7 muestra un comportamiento lineal entre el producto EoWo determinado mediante la ecuación de Stoeckli-Kraehenbuehl con los datos de entalpía experimental y el área accesible, ${ }^{16}$ como lo demuestran los coeficientes de correlación 0,9996 para los discos y 0,9991 para los honeycomb, siendo esto coherente ya que a mayor área se encontró que Wo aumenta, además se puede observar un valor mayor del producto EoWo para las muestras de monolitos tipo disco en comparación con los honeycomb, esto es lógico teniendo en cuenta que los discos presentan una mayor microporosidad y por lo tanto mayor capacidad de adsorción y energía de interacción con un adsorbato.

\section{CONCLUSIONES}

El uso de $\mathrm{ZnCl}_{2}$ como agente impregnante resulta favorable en la síntesis de monolitos de carbón activado utilizando como material precursor cáscara de coco, las estructuras que se obtienen poseen buenas propiedades adsorbentes. Se prepararon 6 muestras de monolitos de carbón (discos y honeycomb), obteniendo áreas BET entre 726 


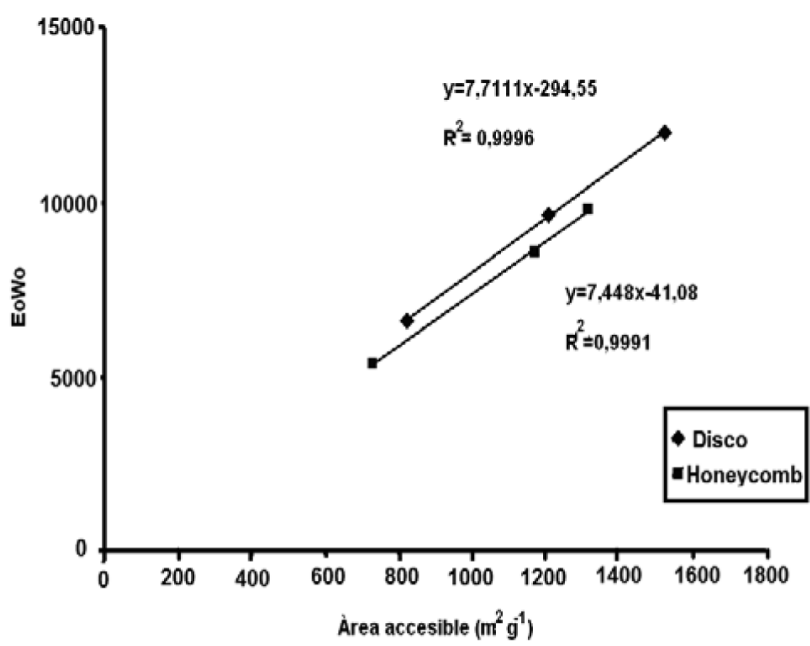

Figura 7. Producto EoWo determinado mediante la ecuación de StoeckliKraehenbuehl en función del área accesible

y $1523 \mathrm{~m}^{2} \mathrm{~g}^{-1}$, y volúmenes de microporo entre 0,38 y $0,79 \mathrm{~cm}^{3} \mathrm{~g}^{-1}$. Las mejores características fueron obtenidas en las muestras MD32 (discos) y MP32 (honeycomb) las cuales se obtuvieron bajo las mismas condiciones, variando únicamente la forma de las estructuras.

Las calorimetrías de inmersión en benceno mostraron una relación directamente proporcional entre el área BET y la entalpía encontrando valores entre 73,5 y 164,2 $\mathrm{J} \mathrm{g}^{-1}$. Se encontró una buena correlación entre el producto EoWo determinado mediante la ecuación de Stoeckli-Kraehenbuehl con los datos de entalpía experimental y el área accesible. Además se observo una cercanía entre los valores de área BET y área accesible, a pesar de que dichos parámetros se determinaron por técnicas de caracterización diferente.

\section{AGRADECIMIENTOS}

Al Convenio Marco entre la Universidad de los Andes y la Universidad Nacional de Colombia y al Acta de Acuerdo entre los
Departamentos de Química de las dos Universidades. Se agradece a las Vicerrectorias de Investigación de la Universidades.

\section{REFERENCIAS}

1. Budinova, T.; Ekinci, E.; Yardim, F.; Grima, A.; Bjornobom, E.; Minkova, V.; Goranova, M.; Fuel Process. Technol. 2006, 87, 899.

2. Laishuan, L.; Zhenyu, L.; Zhanggen, H.; Zenghou, L.; Pingguang, L.; Carbon 2006, 44, 1598.

3. Blanco, J.; Ávila, P.; Martin, M. P.; Microporous Mesoporous Mater. 2000, 37, 201.

4. Gatica, J.; Rodríguez, I. J.; Sánchez, D.; Chafik, T.; Harti, S.; Zaitan, H.; Vidal, H.; C. R. Chim. 2006, 9, 1215.

5. Lozano-Castello, D.; Cazorla-Amorós, D.; Linares-Solano, A.; Quinn, D. F.; Carbon 2002, 40, 2817.

6. Ruiz, V.; Blanco, C.; Santamaría, R.; Ramos, J. M.; Martínezl, M.; Sepúlveda, A.; Rodríguez, F.; Carbon 2009, 47, 195.

7. Rodríguez-Reinoso, F.; Molina-Sabio, M.; Adv. Colloid Interface Sci. 1998, 76-77, 271.

8. Moreno, J. C.; Giraldo, L.; Instrum. Sci. Technol. 2000, 28, 171.

9. Silvestre-Albero, J.; Gómez, C.; Sepúlveda-Escribano, A.; RodríguezReinoso, F.; Colloids Surf., A 2001, 187, 151.

10. Laishuan, L.; Carbon 2006, 44, 1581.

11. Caturla, F.; Molina-Sabio, M.; Rodríguez-Reinoso, F.; Carbon 1991, 29, 999.

12. Molina-Sabio, M.; Almansa, C.; Rodríguez-Reinoso, F.; Spanish pat. 2.165.784, 2003.

13. Almansa, C.; Molina-Sabio, M.; Rodríguez-Reinoso, F.; Microporous Mesoporous Mater. 2004, 76, 185.

14. Nakagawaa, Y.; Molina, M.; Rodríguez, F.; Microporous Mesoporous Mater. 2007, 103, 29.

15. Martín-Martínez, M. J., ed.; Adsorción física de gases y vapores por carbones, Universidad de Alicante (Publicaciones): España, 1990.

16. Moreno, J. C.; Giraldo, L.; Gómez, A.; Instrum. Sci. Technol. 1998, 26, 533. 